\title{
Histerese das isotermas de sorção da polpa de manga (Mangifera indica L.) variedade manteiga
}

\author{
Camila de S. Paglarini' ${ }^{1}$, Fabrício S. da Silva ${ }^{1}$, Alexandre G. Porto ${ }^{1}$, \\ Diego Piasson' ${ }^{1} \&$ Philipe dos Santos ${ }^{2}$
}

\begin{abstract}
RESUMO
As isotermas de sorção são de grande importância para predizer processos de secagem e armazenamento de um produto. A histerese é um fenômeno que ocorre devido à diferença entre as curvas de adsorção e dessorção. O presente trabalho teve o objetivo de analisar a influência da temperatura nas isotermas de sorção e na histerese da polpa de manga variedade manteiga. As isotermas foram determinadas pelo método gravimétrico estático, com a utilização de diferentes concentrações ácidas, nas temperaturas de 20, 30, 40 e $50{ }^{\circ} \mathrm{C}$ e atividade de água variando de 4,3 a $88,8 \%$ até atingir a umidade de equilíbrio. Foram analisados seis modelos matemáticos para isotermas de sorção (GAB, BET, Halsey, Henderson-Thompson, Oswin e Luikov) ajustados aos dados experimentais, através de regressão não-linear. A histerese foi calculada pela área compreendida entre as curvas de dessorção e adsorção. De acordo com os resultados obtidos o modelo de $G A B$ foi o que melhor se ajustou às isotermas de equilíbrio da manga. A área da histerese reduziu $51 \%$ entre as temperaturas de 20 e $50^{\circ} \mathrm{C}$.
\end{abstract}

Palavras-chave: adsorção, dessorção, temperatura, modelo de GAB

\section{Hysteresis of the sorption isotherms of mango pulp (Mangifera indica L.) manteiga variety}

\begin{abstract}
The sorption isotherms are of great importance for predicting drying and storage of a product. Hysteresis is a phenomenon that occurs due to the difference between the curves of adsorption and desorption. This study aimed to analyse the influence of temperature on sorption isotherms and hysteresis of mango pulp manteiga variety. The isotherms were determined by the static method with the use of different acid concentrations. The samples were submitted to temperatures of $20,30,40$ and $50{ }^{\circ} \mathrm{C}$ and water activity ranging from 4.3 to $88.8 \%$ until the equilibrium moisture content. Six mathematical models for the sorption isotherms (GAB, BET, Halsey, Henderson-Thompson, Oswin and Luikov) were analysed and adjusted to the experimental data using nonlinear regression. The hysteresis was calculated as the area between the curves of adsorption and desorption. According to the obtained results, the GAB model was the one that favored the best fit to the equilibrium isotherms of mango. The area of the hysteresis decreased $51 \%$ between temperatures of 20 and $50{ }^{\circ} \mathrm{C}$.
\end{abstract}

Key words: adsorption, desorption, temperature, GAB model

\footnotetext{
' UNEMAT, R. A, s/n, Cohab São Raimundo, C.P. 92, CEP 78390-000, Barra do Bugres, MT. Fone: (65) 3361-1413. E-mail: camilaspaglarini@gmail.com; fabricio@unemat.br; agporto@unemat.br; diegopiasson@yahoo.com.br

2 UNICAMP, R. Monteiro Lobato, 80, CEP 13083-862, Campinas, SP. Fone: (19) 3521-4025. E-mail: philipe.dsn@gmail.com
} 


\section{INTRODUÇÃO}

Nativa do continente asiático, a manga propagou seu cultivo para todos os países da faixa tropical e equatorial do globo. No Brasil a fruta foi amplamente propagada sendo encontradas inúmeras variedades em todas as regiões do país (Franco, 2003). Dados do IBGE (2010) relatam que o Brasil está entre os dez maiores produtores mundiais deste fruto, com produção de 1.188.911 toneladas de manga em 2010.

Com excelentes qualidades nutricionais (carotenóides, carboidratos, fibras e minerais) e característica exótica, a manga se torna uma das principais frutas consumidas no País e de grande importância comercial (Polesi et al., 2011).

A manga variedade manteiga é um fruto pouco explorado, de tamanho médio, casca verde-amarelado e polpa macia, quase sem fibras, além de sabor e odor bastante agradáveis, característicos da manga, possibilitando-lhe grande potencial de industrialização.

Kaymak-Ertekin \& Gedik (2004), Lahsasni et al. (2004) e Fiorentin et al. (2010) estudaram a atividade de água de produtos com alto teor de umidade ( 80 a $90 \%$ ), assim como a manga, uma vez que os dados de atividade de água se tornam indispensáveis nos estudos do processamento deste fruto pois, quanto maior a atividade de água de um produto mais propenso ele estará ao ataque de micro-organismos.

De acordo com Heldman \& Hartel (2000) quando um produto alimentício é submetido a secagem tanto seu teor de umidade como a atividade de água se alteram ao longo do processo devido à relação entre o teor de umidade de equilíbrio do produto e a umidade relativa do ar. Portanto, o estudo desses parâmetros é de grande importância para o desenvolvimento de um processo de secagem haja vista que especifica o teor de umidade do alimento que pode ser atingido sob quaisquer condições do ar de secagem.

Diversos autores estudaram a atividade de água $\left(\mathrm{a}_{\mathrm{w}}\right)$ de determinado produto, através de isotermas de sorção, que representam a relação de equilíbrio entre atividade de água e a umidade de equilíbrio do produto, a temperatura constante (Ahmed et al., 2005; Bellagha et al., 2008).

A base do conhecimento das curvas de adsorção e dessorção é útil para: o processo de secagem, a seleção de um equipamento adequado para secagem, o material da embalagem e a previsão de estabilidade durante a vida útil de armazenamento e transporte do produto (Kaymak-Ertekin \& Gedik, 2004; Samapundo et al., 2007; Tunc \& Duman, 2007).

A isoterma de dessorção possui valores de umidade de equilíbrio superiores aos da isoterma de adsorção a dada atividade de água. A defasagem entre essas duas curvas, denominada histerese, pode ocorrer devido a diversos fatores, tais como condensação capilar, mudanças na estrutura física do material, impurezas na superfície e mudança de fase (Rahman, 1995).

Fellows (2006) descreve que a histerese é importante na determinação da proteção necessária contra o ganho de umidade. Também pode ser usada para estimar a possibilidade de deterioração por reações químicas e micro-organismos (Frankowicz \& Chrenowski, 2006).
Objetivou-se, com este trabalho, analisar a influência da temperatura nas isotermas de sorção e na histerese da polpa de manga variedade manteiga.

\section{Material e Métodos}

O presente estudo foi desenvolvido no Laboratório de Engenharia e Processamento Agroindustrial vinculado ao Centro Tecnológico de Mato Grosso (CTMAT), Campus Universitário Deputado Estadual Renê Barbour, Universidade do Estado de Mato Grosso (UNEMAT).

A matéria-prima utilizada foi a polpa de manga variedade manteiga, extraída de frutos maduros colhidos em pomares nas proximidades do município de Barra do Bugres, MT.

Com vista à determinação das isotermas de dessorção, a manga in natura foi higienizada com solução de hipoclorito de sódio e em seguida imergida em solução de benzoato de sódio a $15 \%$ durante $30 \mathrm{~min}$, com o objetivo de preservar suas características no decorrer do experimento. Para as isotermas de adsorção a polpa foi desidratada em secador descontínuo de bandejas até atingir as condições de equilíbrio e nas de dessorção foi utilizada polpa do fruto in natura, logo após sua obtenção.

Depois, separadamente, as polpas (seca e in natura) foram pesadas, cada unidade com aproximadamente $3 \mathrm{~g}$ e inseridas em recipientes cilíndricos de vidro hermeticamente fechados com $8 \mathrm{~cm}$ de diâmetro e $13 \mathrm{~cm}$ de altura. Em cada recipiente foram adicionados $200 \mathrm{~mL}$ de solução de ácido sulfúrico, para manter a umidade relativa do ar constante no recipiente.

Os recipientes foram colocados em estufas incubadoras com temperatura controlada. Os valores de umidade de equilíbrio foram obtidos no esquema fatorial 4 x 11, com quatro níveis de temperatura $\left(20,30,40\right.$ e $\left.50^{\circ} \mathrm{C}\right)$ e onze níveis de concentrações ácidas $(20,25,30,35,40,45,50,55,60,65$ e 70\%) em triplicata.

Adotou-se o método gravimétrico com determinação do teor de umidade de equilíbrio através do método estático. Este método também foi utilizado por Lahsasni et al. (2004), Bellagha et al. (2008), Pedro et al. (2010), Ansari et al. (2011) e Rangel-Marrón et al. (2011) para determinarem as isotermas de pêra, maçã, maracujá, figo e manga, respectivamente.

Em função das temperaturas e das concentrações ácidas a faixa de atividade de água varia de 4,3 a $88,8 \%$, como pode ser observado na Tabela 1.

Tabela 1. Valores de atividade de água para as concentrações de $\mathrm{H}_{2} \mathrm{SO}_{4}$ para as temperaturas de 20 , 30,40 e $50^{\circ} \mathrm{C}$

\begin{tabular}{ccccc}
\hline Conc. & \multicolumn{4}{c}{ Temperatura $\left.\mathbf{~ (}^{\circ} \mathbf{C}\right)$} \\
\cline { 2 - 5 } $\mathbf{H}_{2} \mathbf{S O}_{4}(\%)$ & $\mathbf{2 0}$ & $\mathbf{3 0}$ & $\mathbf{4 0}$ & $\mathbf{5 0}$ \\
20 & 0,878 & 0,878 & 0,878 & 0,888 \\
25 & 0,816 & 0,817 & 0,824 & 0,829 \\
30 & 0,749 & 0,747 & 0,753 & 0,770 \\
35 & 0,665 & 0,666 & 0,674 & 0,681 \\
40 & 0,568 & 0,565 & 0,574 & 0,582 \\
45 & 0,458 & 0,461 & 0,470 & 0,483 \\
50 & 0,355 & 0,355 & 0,366 & 0,380 \\
55 & 0,258 & 0,260 & 0,267 & 0,280 \\
60 & 0,167 & 0,170 & 0,178 & 0,189 \\
65 & 0,093 & 0,097 & 0,102 & 0,110 \\
70 & 0,043 & 0,045 & 0,049 & 0,055 \\
\hline Fonte: Perry \& Chilton $(1983)$ & &
\end{tabular}


Foram feitas pesagens periódicas em uma balança analítica com precisão de $0,001 \mathrm{~g}$, para verificar se as amostras tinham atingido o equilíbrio higroscópico, ou seja, até que variações de massa entre pesagens se tornassem insignificantes. A primeira pesagem foi realizada em sete dias, a segunda em dez dias e a terceira em quatorze dias, prosseguindo com as pesagens a cada três dias, até atingir o equilíbrio.

Após atingir o equilíbrio as amostras foram submetidas ao método da estufa, $105 \pm 2{ }^{\circ} \mathrm{C}$, até peso constante, para a determinação da umidade de equilíbrio da polpa de manga (IAL, 2008).

Os modelos matemáticos apresentados na Tabela 2 foram ajustados posteriormente aos dados experimentais, mediante análise estatística, por análise de regressão não linear, no software Wolfram Mathematica 8 for sites ${ }^{\circledR}$.

Tabela 2. Principais modelos matemáticos utilizados para descrever isotermas de sorção de alimentos

\begin{tabular}{|c|c|c|c|}
\hline Modelo & & Equação & \\
\hline GAB & $X_{e}=$ & $\frac{\mathrm{X}_{\mathrm{m}} \mathrm{CKa}_{\mathrm{w}}}{\left(1-\mathrm{Ka}_{\mathrm{w}}\right)\left(1-\mathrm{Ka}_{\mathrm{w}}+\mathrm{CKa}_{\mathrm{w}}\right)}$ & (1) \\
\hline Oswin & & $X_{e}=a\left(\frac{a_{w}}{1-a_{w}}\right)^{b}$ & (2) \\
\hline Halsey & & $X_{e}=a\left[T \ln \left(\frac{1}{a_{w}}\right)\right]^{\bar{b}}$ & (3) \\
\hline $\begin{array}{l}\text { Henderson e } \\
\text { Thompson }\end{array}$ & & $x_{e}=\left[\frac{\ln \left(\frac{1}{1-a_{w}}\right)}{a(T+b)}\right]^{\bar{c}}$ & (4) \\
\hline \multirow[t]{2}{*}{ BET } & \multirow{2}{*}{$X_{e}=$} & $\left.\left(x_{m} C a_{w}\right)\left[1-(n+1) a_{w}{ }^{n}+n a_{w}{ }^{n+1}\right]\right]$ & \multirow{2}{*}{ (5) } \\
\hline & & $\left(1-a_{w}\right)\left[1+(c-1) a_{w}-C a_{w}{ }^{n+1}\right]$ & \\
\hline Luikov & & $x_{e}=\frac{a}{1+b T \ln \left(\frac{1}{a_{w}}\right)}$ & (6) \\
\hline
\end{tabular}

$\mathrm{T}$ - temperatura ${ }^{\circ} \mathrm{C} ; \mathrm{X}_{\mathrm{e}}$ - umidade de equilibrio, b.s.; $\mathrm{a}_{\mathrm{w}}$ - Atividade de água, adimensional; $\mathrm{X}_{\mathrm{m}}$ - conteúdo de umidade na monocamada molecular, $\mathrm{kg} \mathrm{kg}^{-1} ; \mathrm{a}, \mathrm{b}, \mathrm{C}, \mathrm{K}$ - constantes de ajuste do modelo; $\mathrm{n}$ - número de camadas moleculares

Os critérios usados para a escolha do melhor ajuste dos modelos aos dados experimentais, foram o coeficiente de determinação $\left(\mathrm{R}^{2}\right)$ e o módulo do desvio médio relativo (P). Utilizou-se também o erro médio estimado (SE) para escolher o melhor ajuste. Os valores de $\mathrm{P}$ e SE foram obtidos pelas seguintes expressões:

$$
\begin{gathered}
P(\%)=\frac{100}{n} \times \sum_{i=1}^{n} \frac{\left[\left(V_{E}-V_{P}\right)\right]}{V_{E}} \\
S E=\sqrt{\frac{\sum_{i=1}^{n}\left(V_{P}-V_{E}\right)^{2}}{n}}
\end{gathered}
$$

em que:

$\mathrm{V}_{\mathrm{E}}$ - valores experimentais

$\mathrm{V}_{\mathrm{P}} \quad$ - valores preditos pelo modelo de regressão não linear

$\mathrm{n} \quad$ - número de dados experimentais

Para calcular a área das curvas de dessorção e adsorção, integrou-se o modelo que melhor se ajustou às isotermas utilizando-se os parâmetros encontrados para o modelo, com o uso dos valores de $\mathrm{a}_{\mathrm{w}}$ determinados por Perry \& Chilton (1983) para cada temperatura estudada.

A histerese foi calculada pela área compreendida entre as curvas de dessorção e adsorção de polpa de manga.

Em relação à dessorção, o Índice de histerese foi adaptado da metodologia utilizada por Picelli et al. (2009) e calculado por meio da Eq. 9:

$$
\frac{\mathrm{AH}}{\mathrm{AD}} \times 100
$$

sendo:

$\mathrm{AH}$ - área da histerese

$\mathrm{AD}$ - área da dessorção.

\section{Resultados E Discussão}

Nas Tabelas 3 e 4 se encontram os valores obtidos experimentalmente representando a umidade de equilíbrio de adsorção e dessorção da polpa de manga manteiga, submetidos nas condições ambientais em soluções ácidas, em função da atividade de água, que variou de 4,3 a $88,8 \%$ e das temperatura de $20,30,40$ e $50^{\circ} \mathrm{C}$.

Tabela 3. Umidade de equilíbrio de adsorção (Xe) da polpa de manga obtida experimentalmente a soluções ácidas e atividade de água $\left(\mathrm{a}_{\mathrm{w}}\right)$ para as temperaturas de $20,30,40$ e $50^{\circ} \mathrm{C}$

\begin{tabular}{cccccccc}
\hline \multicolumn{10}{c}{ Temperatura ('C) } \\
\hline \multicolumn{2}{c}{$\mathbf{2 0}$} & \multicolumn{2}{c}{$\mathbf{3 0}$} & \multicolumn{2}{c}{$\mathbf{4 0}$} & \multicolumn{2}{c}{$\mathbf{5 0}$} \\
\hline $\mathbf{X e}$ & $\mathbf{a}_{\mathbf{w}}$ & $\mathbf{X e}$ & $\mathbf{a}_{\mathbf{w}}$ & $\mathbf{X e}$ & $\mathbf{a}_{\mathbf{w}}$ & $\mathbf{X e}$ & $\mathbf{a}_{\mathbf{w}}$ \\
0,073 & 0,043 & 0,064 & 0,045 & 0,063 & 0,049 & 0,047 & 0,055 \\
0,079 & 0,093 & 0,069 & 0,097 & 0,067 & 0,102 & 0,050 & 0,110 \\
0,087 & 0,167 & 0,077 & 0,170 & 0,071 & 0,178 & 0,052 & 0,189 \\
0,098 & 0,258 & 0,078 & 0,260 & 0,073 & 0,267 & 0,053 & 0,280 \\
0,113 & 0,355 & 0,091 & 0,355 & 0,085 & 0,366 & 0,059 & 0,380 \\
0,135 & 0,458 & 0,112 & 0,461 & 0,104 & 0,470 & 0,087 & 0,483 \\
0,155 & 0,568 & 0,133 & 0,565 & 0,123 & 0,574 & 0,110 & 0,582 \\
0,185 & 0,665 & 0,161 & 0,666 & 0,146 & 0,674 & 0,133 & 0,681 \\
0,240 & 0,749 & 0,204 & 0,747 & 0,198 & 0,753 & 0,173 & 0,770 \\
0,299 & 0,816 & 0,287 & 0,817 & 0,271 & 0,824 & 0,253 & 0,829 \\
0,376 & 0,878 & 0,375 & 0,873 & 0,375 & 0,878 & 0,373 & 0,888 \\
\hline
\end{tabular}

Tabela 4. Umidade de equilíbrio de dessorção (Xe) da polpa de manga obtida experimentalmente a soluções

\begin{tabular}{|c|c|c|c|c|c|c|c|}
\hline \multicolumn{8}{|c|}{ Temperatura $\left({ }^{\circ} \mathrm{C}\right)$} \\
\hline \multicolumn{2}{|c|}{20} & \multicolumn{2}{|c|}{30} & \multicolumn{2}{|c|}{40} & \multicolumn{2}{|c|}{50} \\
\hline $\mathrm{Xe}$ & $a_{w}$ & Xe & $a_{w}$ & Xe & $a_{w}$ & $\mathrm{Xe}$ & $a_{w}$ \\
\hline 0,388 & 0,878 & 0,386 & 0,873 & 0,386 & 0,878 & 0,381 & 0,888 \\
\hline 0,308 & 0,8 & 0,293 & & $\Gamma$ & & 65 & 0,829 \\
\hline 0,248 & 0,7 & 0,212 & & 0,202 & 3 & 5 & 0,770 \\
\hline 0,18 & 0,6 & & & & & & 0 \\
\hline 0,157 & 0,5 & 0,134 & 0,565 & 0,1 & 0,5 & 0,111 & 0,582 \\
\hline 0,1 & & & & & & & \\
\hline 0,13 & 0,3 & y & 5 & 0,099 & 0, & 0,069 & 0,380 \\
\hline 0,12 & 0,2 & & & 0,0 & 0, & & 0,280 \\
\hline 0,11 & & & & 0 & & & 0,189 \\
\hline 0,090 & 0,0 & 0,077 & 0,097 & 0,074 & 0,102 & 0,055 & 0,110 \\
\hline 0,086 & 0,043 & 0,069 & 0,045 & 0,067 & 0,049 & 0,050 & 0,055 \\
\hline
\end{tabular}
ácidas e atividade de água $\left(\mathrm{a}_{\mathrm{w}}\right)$ para as temperaturas de $20,30,40$ e $50^{\circ} \mathrm{C}$ 
Nas Figuras 1A e 1B se apresentam as curvas de isotermas de adsorção e dessorção da polpa de manga Manteiga, respectivamente, para todas as temperaturas estudadas.

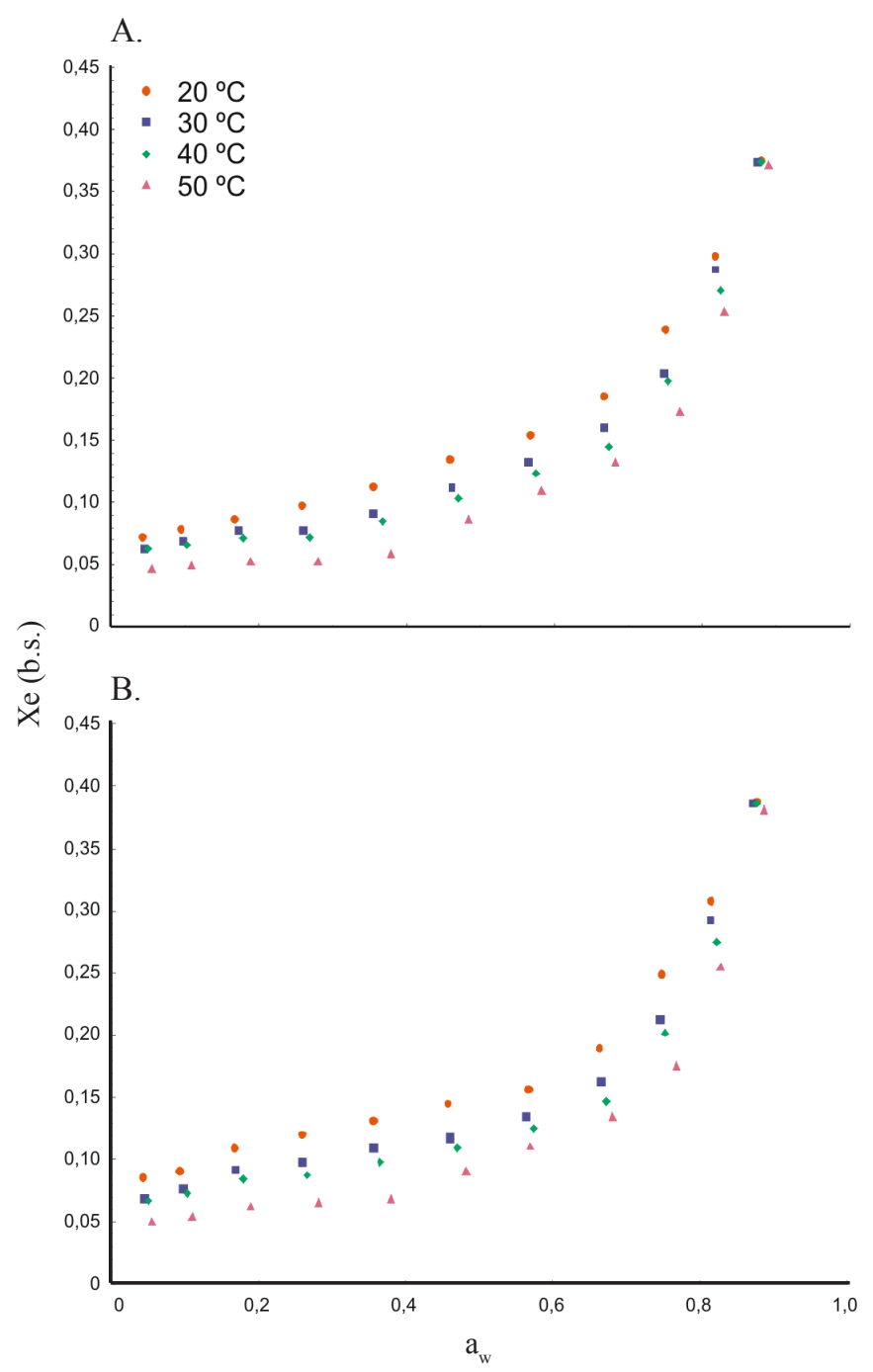

Figura 1. Isotermas de adsorção (A) e dessorção (B) da polpa de manga Manteiga nas temperaturas 20, 30,40 e $50{ }^{\circ} \mathrm{C}$

Conforme as Figuras 1A e 1B, as isotermas de equilíbrio da polpa de manga evidenciaram curvas com comportamento sigmoidal, do tipo II, de acordo com a classificação de Brunauer et al. (1940); segundo Medeiros et al. (2006) esta é a forma de isoterma mais comum em alimentos. Gabas et al. (2009) também observaram este comportamento em seu estudo.

Os modelos matemáticos foram ajustados aos dados de umidade de equilíbrio para que fosse escolhido o modelo que melhor representasse tal fenômeno observando-se, para tanto, os conteúdos de umidade em função da variação de temperatura em cada experimento.

Nas Tabelas 5 e 6 se encontram os parâmetros determinados para os modelos, testados e ajustados para a polpa de manga em função das atividades de água e das temperaturas estudadas para cada uma das equações tal como, também, os coeficientes de determinação $\left(\mathrm{R}^{2}\right)$ e desvios médios relativos $(\mathrm{P})$ e erros médios estimado (SE).
Como se observa nas Tabelas 5 e 6, o modelo de GAB descreveu com maior precisão as isotermas de sorção para a polpa de manga, fazendo-se uso de soluções ácidas pois possui valores de $\mathrm{R}^{2}$ próximos de 1 , valores de $\mathrm{P}$ menores que $10 \% \mathrm{e}$ valores de SE próximos de zero, porém o modelo de Halsey também ajustou satisfatoriamente essas isotermas.

De acordo com Pedro et al. (2010) o modelo de GAB apresenta a vantagem de ser um modelo relativamente simples e com parâmetros que têm definição física, além de representar adequadamente os dados experimentais na faixa de atividade de água de maior interesse prático em alimentos $(0,10$ a 0,90$)$. Lahsasni et al. (2004), Oliveira et al. (2005) e Medeiros et al. (2006) obtiveram bom ajuste pelo modelo de GAB para isotermas de pera, casca de abacaxi, cacau e cupuaçu, respectivamente.

Observa-se, nas Tabelas 5 e 6 , que os valores da monocamada de GAB variaram de 0,083 a $0,045 \mathrm{~kg} \mathrm{~kg}^{-1}$, de 20 para $50{ }^{\circ} \mathrm{C}$, ou seja, reduziram com o aumento da temperatura. Prado et al. (1999) relatam que o valor de $X_{m}$ diminui consideravelmente com o aumento da temperatura devido à redução de número de zonas ativas como resultado de mudanças físicas e/ou químicas induzidas pela temperatura.

Segundo Gabas et al. (2009) o valor de umidade de monocamada $\left(\mathrm{X}_{\mathrm{m}}\right)$ é de grande interesse visto que indica a quantidade de água que é fortemente adsorvida em locais específicos do alimento, considerado o melhor valor para garantir sua estabilidade.

Visualiza-se também, nas Tabelas 5 e 6 , que os valores da monocamada são maiores na dessorção $\left(0,045\right.$ a $\left.0,083 \mathrm{~kg} \mathrm{~kg}^{-1}\right)$ do que na adsorção $\left(0,042\right.$ a $\left.0,076 \mathrm{~kg} \mathrm{~kg}^{-1}\right)$.

As constantes $\mathrm{C}$ e $\mathrm{K}$ de $\mathrm{GAB}$ são um indicativo do tipo de isoterma, de acordo com a classificação de Brunauer et al. (1938). Observando os parâmetros C e K nas Tabelas 5 e 6, constata-se que em todas as temperaturas $\mathrm{K}<1$ e $\mathrm{C}>2$ o que, de acordo com Blahovec (2004) para esses valores as isotermas são do Tipo II, conforme verificado visualmente nas Figuras $1 \mathrm{~A}$ e $1 \mathrm{~B}$.

Nas Figuras 2A, 2B, 2C e 2D é apresentada a histerese para a polpa de manga manteiga, nas temperaturas de 20,30, 40 e $50{ }^{\circ} \mathrm{C}$, ajustadas pelo modelo de $\mathrm{GAB}$.

As isotermas de equilíbrio apresentadas nas Figuras 2A a 2D, mostram que a curva de adsorção está abaixo da curva de dessorção em todo o intervalo de atividade de água e nas quatro temperaturas consideradas. Segundo Ayrosa (2005) isto ocorre devido ao fenômeno da histerese que faz com que seja necessária uma pressão menor de vapor para se atingir certo teor de umidade por um processo de dessorção que por adsorção.

Caurie (2007) relata que a histerese pode ser utilizada como índice de qualidade alimentar uma vez que seu aumento indica redução da estabilidade do alimento; já sua redução ou ausência indica melhor estabilidade dos produtos armazenados.

Na Figura 2 pode-se observar que o efeito da histerese para a polpa de manga manteiga reduziu com o aumento da temperatura; Damodaran et al. (2010) relatam que este fenômeno é mais evidente a baixas temperaturas.

A Tabela 7 mostra os resultados obtidos pela integração entre as curvas de dessorção e adsorção através do modelo de 
Tabela 5. Parâmetros de ajuste das isotermas de adsorção em polpa de manga submetida a soluções ácidas para os diferentes modelos matemáticos

\begin{tabular}{|c|c|c|c|c|c|c|c|c|c|c|}
\hline \multirow{2}{*}{ Modelo } & \multirow{2}{*}{$\begin{array}{c}\mathrm{T} \\
\left({ }^{\circ} \mathrm{C}\right)\end{array}$} & \multicolumn{9}{|c|}{ Parâmetros } \\
\hline & & A & B & C & $\overline{X_{m}}$ & $\mathrm{~N}$ & K & $\mathbf{R}^{2}$ & $\mathbf{P}(\%)$ & SE \\
\hline \multirow{4}{*}{ GAB } & 20 & & & 267,4 & 0,076 & & 0,910 & 0,9994 & 0,077 & 0,00314 \\
\hline & 30 & & & 3865057 & 0,060 & & 0,963 & 0,9989 & 1,024 & 0,00454 \\
\hline & 40 & & & 31934501 & 0,054 & & 0,974 & 0,9988 & 2,376 & 0,00520 \\
\hline & 50 & & & 925820,9 & 0,042 & & 0,998 & 0,9982 & 0,560 & 0,00598 \\
\hline \multirow{4}{*}{ BET } & 20 & & & 0,00426 & 51,490 & 1,441 & & 0,9447 & 17,535 & 0,03812 \\
\hline & 30 & & & 0,00243 & 43,981 & 2,360 & & 0,9491 & 23,945 & 0,03904 \\
\hline & 40 & & & 0,00203 & 37,771 & 2,926 & & 0,9506 & 27,009 & 0,03950 \\
\hline & 50 & & & 0,00128 & 30,555 & 4,594 & & 0,9714 & 33,096 & 0,03261 \\
\hline \multirow{4}{*}{ Halsey } & 20 & 0,6646 & 1,7034 & & & & & 0,9981 & 0,695 & 0,03537 \\
\hline & 30 & 0,9417 & 1,4885 & & & & & 0,9940 & 4,037 & 0,01102 \\
\hline & 40 & 1,1502 & 1,4250 & & & & & 0,9919 & 5,351 & 0,01281 \\
\hline & 50 & 1,5421 & 1,2352 & & & & & 0,9950 & 6,215 & 0,01091 \\
\hline \multirow{4}{*}{ Luikov } & 20 & 0,5531 & 0,2047 & & & & & 0,9887 & 1,801 & 0,09518 \\
\hline & 30 & 0,7142 & 0,2420 & & & & & 0,9866 & 11,711 & 0,01939 \\
\hline & 40 & 0,8039 & 0,2391 & & & & & 0,9858 & 13,484 & 0,02029 \\
\hline & 50 & 1,2959 & 0,4300 & & & & & 0,9930 & 13,434 & 0,01470 \\
\hline \multirow{4}{*}{ Oswin } & 20 & 0,1503 & 0,4492 & & & & & 0,9884 & 7,145 & 0,01632 \\
\hline & 30 & 0,1283 & 0,5260 & & & & & 0,9807 & 8,855 & 0,02043 \\
\hline & 40 & 0,1160 & 0,5594 & & & & & 0,9781 & 16,910 & 0,03176 \\
\hline & 50 & 0,0882 & 0,6737 & & & & & 0,9870 & 13,698 & 0,01801 \\
\hline \multirow{4}{*}{ Henderson e Thompson } & 20 & 0,2060 & 30,947 & 1,485 & & & & 0,9657 & 9,077 & 0,02665 \\
\hline & 30 & 0,2430 & 0,565 & 1,171 & & & & 0,9599 & 14,296 & 0,03042 \\
\hline & 40 & 0,1700 & 0,187 & 1,066 & & & & 0,9573 & 16,910 & 0,03176 \\
\hline & 50 & 0,1050 & 0,106 & 0,815 & & & & 0,9749 & 23,880 & 0,02685 \\
\hline
\end{tabular}

Tabela 6. Parâmetros de ajuste das isotermas de dessorção em polpa de manga submetida a soluções ácidas para os diferentes modelos matemáticos

\begin{tabular}{|c|c|c|c|c|c|c|c|c|c|c|}
\hline \multirow{2}{*}{ Modelo } & \multirow{2}{*}{$\begin{array}{c}\mathrm{T} \\
\left({ }^{\circ} \mathrm{C}\right)\end{array}$} & \multicolumn{9}{|c|}{ Parâmetros } \\
\hline & & A & B & C & $\bar{X}_{m}$ & $\mathbf{N}$ & K & $\mathbf{R}^{2}$ & $\mathbf{P ( \% )}$ & SE \\
\hline \multirow{4}{*}{ GAB } & 20 & & & 1234660 & 0,083 & & 0,891 & 0,9957 & 1,845 & 0,00888 \\
\hline & 30 & & & 2578480 & 0,065 & & 0,949 & 0,9958 & 3,107 & 0,00929 \\
\hline & 40 & & & 2873436 & 0,058 & & 0,965 & 0,9953 & 3,700 & 0,01021 \\
\hline & 50 & & & 2066197 & 0,045 & & 0,993 & 0,9983 & 1,650 & 0,00607 \\
\hline \multirow{4}{*}{ BET } & 20 & & & 0,0051 & 79,423 & 0,915 & & 0,9015 & 12,075 & 0,04513 \\
\hline & 30 & & & 0,0029 & 52,912 & 1,849 & & 0,9203 & 20,408 & 0,04534 \\
\hline & 40 & & & 0,0023 & 41,953 & 2,382 & & 0,9231 & 27,693 & 0,04649 \\
\hline & 50 & & & 0,0018 & 24,087 & 4,350 & & 0,9621 & 33,039 & 0,03722 \\
\hline \multirow{4}{*}{ Halsey } & 20 & 0,625 & 1,922 & & & & & 0,9926 & 1,833 & 0,01142 \\
\hline & 30 & 0,895 & 1,593 & & & & & 0,9887 & 4,113 & 0,01481 \\
\hline & 40 & 1,187 & 1,409 & & & & & 0,9886 & 11,13 & 0,01773 \\
\hline & 50 & 1,474 & 1,282 & & & & & 0,9922 & 7,465 & 0,01313 \\
\hline \multirow{4}{*}{ Luikov } & 20 & 0,499 & 0,149 & & & & & 0,9705 & 8,1125 & 0,02553 \\
\hline & 30 & 0,650 & 0,194 & & & & & 0,9759 & 12,056 & 0,02518 \\
\hline & 40 & 0,872 & 0,265 & & & & & 0,9824 & 18,848 & 0,02630 \\
\hline & 50 & 1,189 & 0,375 & & & & & 0,9898 & 15,691 & 0,01824 \\
\hline \multirow{4}{*}{ Oswin } & 20 & 0,169 & 0,388 & & & & & 0,9752 & 4,392 & 0,02130 \\
\hline & 30 & 0,140 & 0,482 & & & & & 0,9706 & 7,835 & 0,02443 \\
\hline & 40 & 0,116 & 0,568 & & & & & 0,9736 & 15,838 & 0,02671 \\
\hline & 50 & 0,094 & 0,644 & & & & & 0,9818 & 14,040 & 0,02105 \\
\hline \multirow{4}{*}{ Henderson e Thompson } & 20 & 0,150 & 71,778 & 1,732 & & & & 0,9438 & 6,4195 & 0,03212 \\
\hline & 30 & 0,289 & 0,747 & 1,329 & & & & 0,9411 & 11,469 & 0,03503 \\
\hline & 40 & 0,193 & 0,322 & 1,183 & & & & 0,9386 & 14,079 & 0,03638 \\
\hline & 50 & 0,112 & 0,106 & 0,874 & & & & 0,9642 & 22,103 & 0,03078 \\
\hline
\end{tabular}

GAB definindo, assim, a área de histerese de acordo com o procedimento utilizado por Picelli et al. (2009).

Verificando a Tabela 7, constatou-se uma área maior para as isotermas de dessorção do que de adsorção comprovando, deste modo, o que está escrito na literatura, ou seja, a curva de dessorção sempre acima da curva de adsorção para todas as temperaturas estudadas.
A área da histerese comportou-se de forma inversamente proporcional à temperatura, isto é, de 20 para $50^{\circ} \mathrm{C}$ esta área reduziu cerca de $51 \%$. Yan et al. (2008) também encontraram, ao trabalhar com banana, redução da área da histerese com o aumento da temperatura.

Observa-se também que, para $20^{\circ} \mathrm{C}$, o índice de histerese em relação à dessorção (IHRD) foi de $6,25 \%$ e para $50^{\circ} \mathrm{C}$ houve 
A.

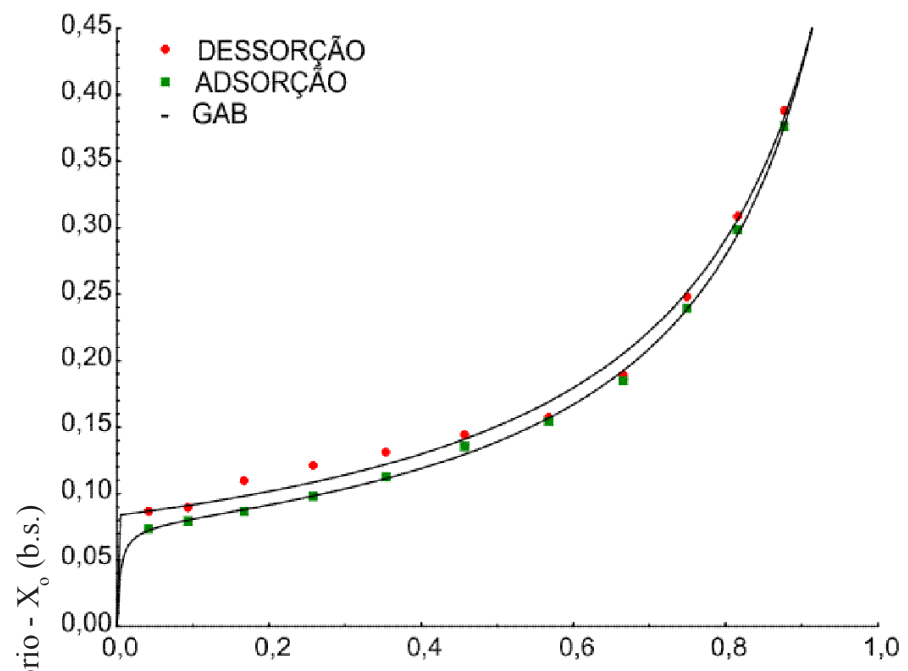

C.

으 0,45

兽

0,40
0

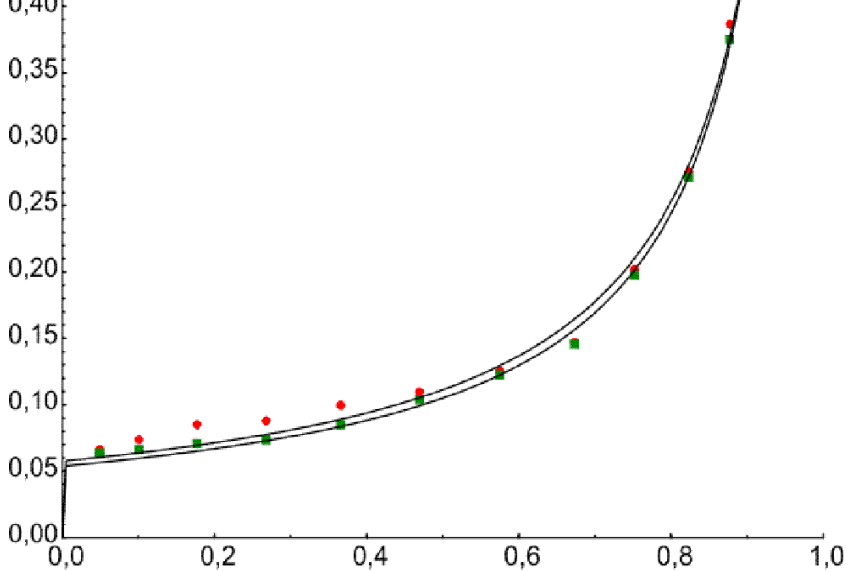

B.

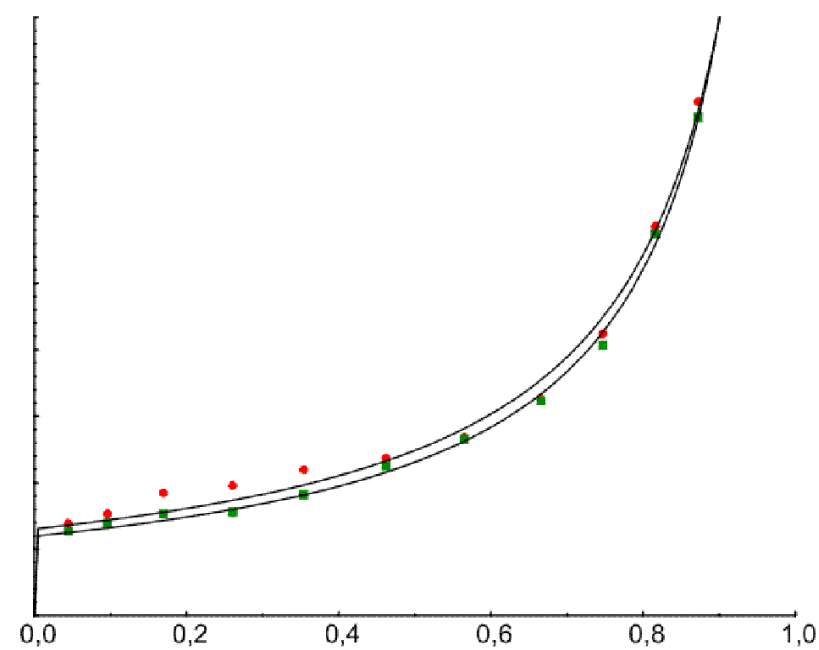

D.

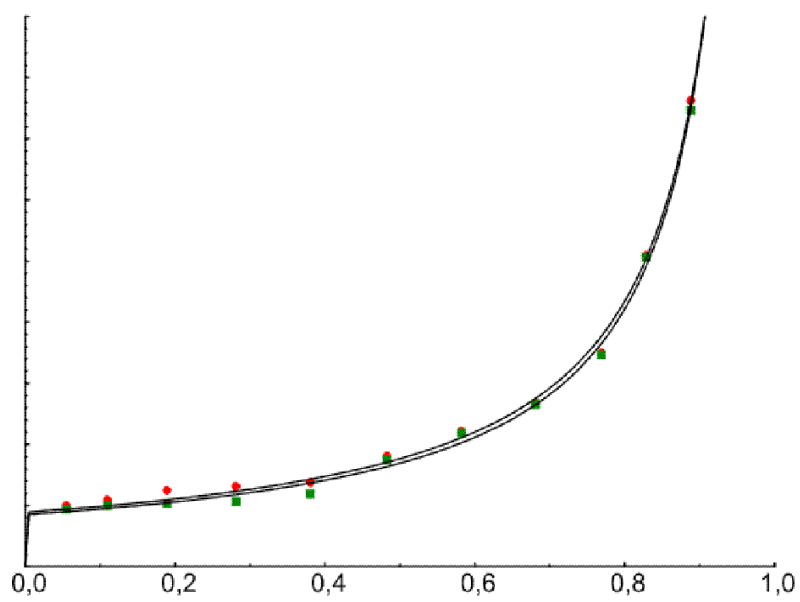

Atividade da água $\left(\mathrm{a}_{\mathrm{w}}\right)$

Figura 2. Histerese para as isotermas de equilíbrio da polpa de manga para a temperatura de 20 (A), 30 (B), 40 (C) e $50{ }^{\circ} \mathrm{C}(\mathrm{D})$ ajustadas pelo modelo de $\mathrm{GAB}$

Tabela 7. Histerese encontrada para isoterma de sorção de polpa de manga nas temperaturas estudadas

\begin{tabular}{ccccc}
\hline Temperatura & \multicolumn{3}{c}{ Área } & \multirow{2}{*}{ IHRD* } \\
\cline { 2 - 4 }$\left({ }^{(} \mathbf{C}\right)$ & Dessorção & Adsorção & Histerese & \\
20 & 0,138385 & 0,129738 & 0,00864777 & $6,25 \%$ \\
30 & 0,136326 & 0,127725 & 0,00748799 & $5,49 \%$ \\
40 & 0,137866 & 0,129299 & 0,00580839 & $4,21 \%$ \\
50 & 0,141237 & 0,132720 & 0,00427450 & $3,03 \%$ \\
\hline
\end{tabular}

* Índice de Histerese em Relação à Dessorção

redução para 3,03\% demonstrando a influência da temperatura neste efeito.

\section{Conclusões}

1. O modelo de GAB foi o que descreveu com maior precisão as isotermas de adsorção e dessorção da polpa de manga variedade manteiga.

2. A temperatura teve influência na área da histerese de polpa de manga.
3. Demonstrou-se a diminuição do fenômeno de histerese com o aumento da temperatura.

4. A área da histerese reduziu $51 \%$ entre as temperaturas de 20 e $50^{\circ} \mathrm{C}$.

5. O índice de histerese em relação a dessorção (IHRD) foi de $6,25 \%$ para $20{ }^{\circ} \mathrm{C}$ e $3,03 \%$ para $50{ }^{\circ} \mathrm{C}$.

\section{Agradecimentos}

A equipe executora deste trabalho agradece ao Conselho Nacional de Desenvolvimento Científico e Tecnológico-CNPq, pelas bolsas concedidas, e à Fundação de Amparo à Pesquisa do Estado de Mato Grosso - FAPEMAT, pelo auxílio financeiro.

\section{Literatura Citada}

Ahmed, J.; Ranaswamy, H.S.; Khan, A.R. Effect of water activity on glass transitions of date pastes. Journal of Food Engineering, v.66, p.253-258, 2005. 
Ansari, S.; Farahnaky, A.; Majzoobi, M. Modeling the effect of glucose syrup on the moisture sorption isotherm of figs. Food Biophysics, v.6, p.377-389, 2011.

Ayrosa, A. M. I. B. Atividade de água e suas aplicações nos processos de conservação, secagem e manipulação de alimentos e medicamentos. Revista de Engenharia FAAP, v.18, p.41-47, 2005.

Bellagha, S.; Sahli, A.; Benzid, M.; Farhat, A. Desorption isotherms of fresh and osmotically dehydrated apples (Golden delicious). Revue des Energies Renouvelables, n.2, p.45-52, 2008.

Blahovec, J. Sorption isotherms in materials of biological origin mathematical and physical approach. Journal of Food Engineering, v.65, p.489-495, 2004.

Brunauer, S.; Deming, L. S.; Teller, E. On a theory of Van der Waals adsorption of gases. Journal of the American Chemical Society, v.62, p.1723-1732, 1940.

Caurie, M. Hysteresis phenomenon in foods. International Journal of Food Science and Technology, v.42, p.45-49, 2007.

Damodaran, S.; Parkin, K. L.; Fennema, O. R. Química de Alimentos de Fennema. 4.ed., Porto Alegre: Artmed, 2010. 900p.

Fellows, J. P. Tecnologia do processamento de Alimentos: Princípios e Prática. 2.ed., Porto Alegre: Artmed, 2006. $602 p$.

Fiorentin, L. D.; Menon, B. T.; Barros, S. T. D.; Pereira, N. C.; Lima, O. C. M.; Modenes, A. N. Isotermas de sorção do resíduo agroindustrial bagaço de laranja. Revista Brasileira de Engenharia Agrícola e Ambiental, v.14, p.653-659, 2010.

Franco, M. R. B. Aroma e sabor de alimentos: temas atuais. São Paulo: Livraria Varela, 2003. 246p.

Frankowicz, M.; Chrenowski, M. Application of Preisach model to adsorption - desorption hysteresis. Physica B, v.372, p.219-221, 2006.

Gabas, A. L.; Telis-Romero, J.; Giraldo-Gómez, G. I.; Telis, V. R. N. Propiedades termodinámicas de sorción de agua de la pulpa de lulo en polvo com encapsulantes. Ciências e Tecnologia de Alimentos, v.29, p.911-918, 2009.

Heldman, D.R.; Hartel, R.W. Principles of food processing. New York: Chapman \& Hall, 2000. 218p.

IAL - Instituto Adolfo Lutz. Normas Analíticas do Instituto Adolfo Lutz: Métodos químicos e físicos para análise de alimentos. São Paulo: IMESP, 4.ed., 2008. 1020p.

IBGE - Instituto Brasileiro de Geografia e Estatística. Diretoria de Pesquisas, Coordenação de Agropecuária, Produção Agrícola Municipal. Rio de Janeiro: Gráfica Digital, v.37. 2010. 91p.

Kaimak-Ertekin, F.; Gedik, A. Sorption isotherms and isosteric heat of sorption for grapes apricots, apples and potatoes. Lebensmittel - Wissenschaft und Technologie, v.37, p.429$438,2004$.
Lahsasni, S.; Kouhila, M.; Mahrouz, M. Adsorption-desorption isotherms and heat of sorption of prickly pear fruit (Opuntia ficus indica). Energy Conversion and Management, v.45, p.249-26, 2004.

Medeiros, M. L.; Ayrosa, A. M. I. B.; Pitombo, R. N. M.; Lannes, S. C. S. Sorption isotherms of cocoa and cupuassu products. Journal of Food Engineering, v.73, p.402-406, 2006.

Oliveira, M. M.; Campos, A. R. N.; Gomes, J. P.; Silva, F. L. H. da. Isotermas de sorção do resíduo agroindustrial de casca do abacaxi (Ananas comosus L. Mer.). Revista Brasileira de Engenharia Agrícola e Ambiental, v.9, p.565-569, 2005.

Pedro, M. A. M.; Telis-Romero, J.; Telis, V. R. N. Effect of drying method on the adsorption isotherms and isosteric heat of passion fruit pulp powder. Ciência e Tecnologia de Alimentos, v.30, p.993-1000, 2010.

Perry, R. H.; Chilton, C. H. Chemical engineer's handbook. New York: McGraw-Hill Co., 5.ed., 1983, p.20-24.

Picelli, R. M.; Arrieche, L.S.; Sartori, D. J. M. Histerese de isotermas de adsorção e dessorção de umidade de equilíbrio para sementes de embaúba. In: Simpósio Internacional de Iniciação Científica, 17, 2009, São Carlos. Anais ...USP, 2009, v.1, p.2-2.

Polesi, L. F.; Matta Júnior, M. D. da; Matsuoka, C. R.; Ceballos, C. H. de M.; Anjos, C. B. P. dos; Spoto, M. H. F.; Sarmento, S. B. S. Caracterização química e física de geléia de manga de baixo valor calórico. Revista Brasileira de Produtos Agroindustriais, v.13, p.85-90, 2011.

Prado, M. E. T.; Alonso, L. F. T.; Sales, A. F.; Park, K. J. Isotermas de sorção de tâmaras: Determinação experimental e avaliação de modelos matemáticos. Ciência e Tecnologia de Alimentos, v.19, p.143-146, 1999.

Rahman, M. S. Food Properties Handbook. Boca Raton: CRC Press, 1995. 86p.

Rangel-Marrón M.; Welti-Chanes J.; Córdova-Quiroz A.V.; Cerón-Bretón J.G.; Cerón-Bretón R. M.; AnguebesFranseschi F. Estimation of moisture sorption isotherms of mango pulp freeze- dried. International Journal of Biology and Biomedical Engineering, v.5, p.18-23, 2011.

Samapundo, S.; Devlieghere, F.; Meulenaer, B. D.; Tukwase, A.; Lamboni.Y.; Debevere, J.M. Sorption isotherms and isosteric heats of sorption of whole yellow dent corn. Journal of Food Engineering, v.79, p.168-175, 2007.

Tunc, S.; Duman, O. Thermodynamic properties and moisture adsorption isotherms of cottonseed protein isolate and different forms of cottonseed samples. Journal of Food Engineering, v.81, p.133-143, 2007.

Yan, Z.; Sousa-Gallagher, M. J.; Oliveira, F. A. R. Sorption isotherms and moisture sorption hysteresisof intermediate moisture content banana. Journal of Food Engineering, v.86, p.342-348, 2008. 